\title{
Intención de compra de servicios de alojamiento a través de redes sociales: aplicación del modelo de aceptación tecnológica
}

\section{Intention to purchase accommodation services through social networks: application of the technological acceptance model}

Carlos Aníbal Manosalvas Vaca

Universidad Estatal Amazónica, Ecuador

cmanosalvas@uea.edu.ec

https://orcid.org/0000-0002-7521-069X

Yolanda Lorena Paredes Andrade

Universidad Estatal Amazónica, Ecuador

yparedes@uea.edu.ec

https://orcid.org/0000-0001-9764-2779

Recepción: 29/12/2020 | Aceptación: 03/03/2021 | Publicación: 10/05/2021

Cómo citar (APA, séptima edición):

Manosalvas Vaca, C. A. y Paredes Andrade, Y. L. (2021). Intención de compra de servicios de alojamiento a través de redes sociales: aplicación del modelo de aceptación tecnológica. Innova Research Journal, 6(2), 274-281. https://doi.org/10.33890/innova.v6.n2.2021.1630

\section{Resumen}

Las tecnologías de la información y comunicación han generado una gran influencia en los ámbitos empresariales, así como en el comportamiento de los consumidores. Específicamente en el sector turístico, los clientes tienen la posibilidad de acceder a gran cantidad de oferta de servicios lo cual obliga a las empresas a identificar los factores a través de los cuales pueden captar y mantener la atención y comunicación con el cliente con el propósito de incrementar las ventas de sus servicios. Con base en esto, en esta investigación se analizó si la utilidad percibida y la facilidad de uso percibida, que son componentes del modelo de aceptación tecnológico, influyen en la intención de compra de servicios de alojamiento a través de sitios de redes sociales. La investigación aplicada a una muestra de 382 turistas, aplicando un enfoque cuantitativo haciendo uso de modelos de ecuaciones estructurales (SEM), se evidenció que únicamente la facilidad de uso percibida influye en la intención de compra online de servicios de alojamiento. Los resultados permiten concluir que es necesario poner más énfasis en los aspectos hedónicos de estas plataformas virtuales.

Palabras claves: aceptación tecnológica; intención de compra; redes sociales. 


\begin{abstract}
The information and communication technologies have generated a great influence in business environments, as well as in consumer behavior. Specifically, in the tourism sector, clients have the possibility of accessing a large amount of service offerings, which forces companies to identify the factors through which they can capture and maintain customer service and communication with the purpose of increase the sales of your services. Based on this, this research analyzed whether perceived utility and perceived ease of use, which are components of the technology acceptance model, influence the intention to purchase accommodation services through social networking sites. The research applied to a sample of 382 tourists and making use of structural equation modeling, showed that only the perceived ease of use influences the intention to buy online. The results allow us to conclude that it is necessary to put more emphasis on the hedonic aspects of these virtual platforms.
\end{abstract}

Keywords: technological acceptance; purchase intent; social networks.

\title{
Introducción
}

El acelerado crecimiento de las Tecnologías de la Información y comunicación han generado cambios importantes en todos los ámbitos del quehacer humano, principalmente, en las actividades empresariales. De todas las tecnologías que se han creado, el e-commerce o la actividad de compra o venta de artículos o servicios online, se ha extendido y tiene amplia aplicación en diferentes industrias y empresas de distintos tamaños. En el sector turístico y específicamente en el sector de alojamiento, estos cambios son más evidentes puesto que, en la actualidad, los turistas utilizan diferentes canales electrónicos para buscar y comprar servicios de hospedaje los cuales incluyen sitios web y redes sociales.

De acuerdo a Aluri (2012) el turismo es una de las industrias líderes que utilizan aplicaciones basadas en la web para llegar a los usuarios; sin embargo, a diferencia de los clientes tradicionales, los usuarios que utilizan Internet pueden encontrar fácilmente un mejor producto o servicio en línea. Por lo tanto, su poder de conmutación es alto lo que hace imperativo analizar de qué manera se puede mantener su permanencia en el sitio web de la empresa. Una estrategia para lograrlo es el uso de redes sociales, aplicaciones que emergieron debido a la evolución de la tecnología Web 1.0 hacia la Web 2.0 (Hajli, 2015).

La creciente popularidad de los sitios de redes sociales ha generado varios cambios principalmente en la interacción entre los consumidores y las empresas, originando nuevas formas de realizar transacciones en línea (Busalim, Chen Hussin y Fadhil Yusof, 2017). Los usuarios emplean estas aplicaciones como una herramienta de colaboración para compartir experiencias de compra en línea e información relacionada con productos y servicios; además brinda la posibilidad de obtener los mejores precios intercambiando información confiable sobre ciertos productos y servicios (Kim y Park, 2013). Estos aspectos hacen imprescindible analizar qué factores influyen en la intención de compra de servicios de turísticos y, específicamente de alojamiento, a través de redes sociales. Como lo manifestaron, Law y Chang (2006), en un entorno donde las empresas tienen que competir por turistas que busca información en línea, comprender sus necesidades y los 
factores que influyen en sus intenciones de compra son esenciales para el éxito de las empresas hoteleras.

Con base en esto, en la presente investigación se utiliza el Modelo de Aceptación Tecnológica (TAM por sus siglas en inglés) para analizar la relación de la Facilidad de Uso Percibida y la Utilidad percibida de las redes sociales con la Intención de Compra de servicios de alojamiento. De acuerdo a este modelo, la voluntad de las personas de usar o no usar una tecnología específica depende de la eficiencia de ese método para mejorar el resultado de un comportamiento específico, que se define como utilidad mientras que la facilidad de uso se define como el grado en que una persona cree que confiar en un sistema en particular sería fácil (Davis, 1989).

\section{Revisión de la Literatura}

\section{Modelo de Aceptación Tecnológica TAM}

El modelo de aceptación tecnológica TAM fue propuesto por Davis (1989) y fue desarrollado tomando como preceptos la Teoría de la Acción Razonada (TRA) propuesta por Fishbein y Ajzen (1975) y la Teoría del Comportamiento Planificado (TPB) propuesta por Ajzen (1991). Este modelo incluye dos componentes específicos de comportamiento: (a) utilidad percibida y (b) facilidad de uso percibida; componentes que determinan el comportamiento de un individuo relacionado a la intención de uso de una tecnología.

Según Yilaz (2014) TAM explica que, la decisión del usuario de usar un nuevo dispositivo técnico o paquete de software está determinada por la intención de comportamiento de usar el sistema. Esta intención de comportamiento está influenciada por la facilidad de uso percibida (PEOU) del individuo del sistema y su utilidad percibida (PU) (Chu y Chu, 2011). La utilidad percibida se refiere al grado en que un individuo cree que usar una tecnología en particular mejoraría su desempeño laboral. La facilidad de uso percibida se refiere al grado en que un individuo cree que usar una tecnología en particular estaría libre de esfuerzo físico y mental (Wallace y Sheetz, 2014). TAM es uno de los modelos más utilizados para investigar la adopción de una nueva tecnología ya que es considerada una teoría parsimoniosa y poderosa por la comunidad académica dedicada a estudiar los diferentes aspectos de los Sistemas de Información.

\section{Intención de Compra en Línea}

Comprar es una experiencia social y las redes sociales permiten a los consumidores interactuar (Pookulangara y Koesler, 2011), lo que ha provocado que los consumidores actuales utilizan cada vez más la tecnología como una herramienta eficaz para comprar en plataformas en línea (Ramayah, Rahman y Ling, 2018), un hecho que ha impulsado el crecimiento del comercio basado en Internet en todo el mundo, puesto que es conveniente y apropiado para los consumidores (Javed, Nazam, Ahmad, Nadeem y Qadeer, 2015). Además, la aceptación de la Web 2.0 ha abierto la puerta a la construcción de un nuevo mundo de colaboración y comunicación (Ramayah et. al., 2018). 
Pavlou (2003) definió la intención de compra en línea como una situación en la que un consumidor está dispuesto y tiene la intención de realizar transacciones en línea. Anteriormente, Zwass (1998) definió la intención de compra en línea como la intención del consumidor de crear una asociación en línea y realizar transacciones con un proveedor web. Pavlou (2003) identificó tres pasos secuenciales que juntos componen una transacción en línea: (a) recuperación de información, (b) transferencia de información y (c) compra de productos. Sin embargo, San Martín y Herrero (2012) sugirieron que la intención de compra en línea también puede considerarse como un indicador confiable del grado de reconocimiento de las nuevas tecnologías por parte de los usuarios.

\section{Modelo de Aceptación tecnológica (TAM) en la Intención de Compra en Línea}

Un sitio web es en esencia una tecnología de información y como tal, la intención de compra en línea a través de estos sitios debería ser explicada en parte por el modelo de aceptación tecnológica (Gefen, Karahanna y Straub, 2003). Davis (1989) argumentó que la Facilidad de Uso Percibida y la Utilidad Percibida influyen conjuntamente en las intenciones de comportamiento. Kim y Song (2006) proponen que la utilidad percibida está relacionada con la actitud de compra entre los compradores en línea. Si los consumidores encuentran útil un sitio web de comercio electrónico para comprar, tendrán una mejor actitud de compra electrónica. Otros estudios como los de Yoon y Steege (2013); Punnoose (2012); Aldás-Manzano, Ruiz-Mafé y Sanz-Blas (2009); Özbek, Alnıaçık, Koc, Akkılıç, y Kaş (2014); y Devaraj, Esaley y Crant (2008) también confirman la relación entre Utilidad Percibida e Intención de Compra.

Por otro lado, varios investigadores han encontrado una relación positiva entre el Facilidad de uso percibida e Intención de Compra. Childers, Carr, Peck y Carson (2001) sugieren que los sitios de compras en línea claros y comprensibles, que requieren menos esfuerzos mentales de sus usuarios para realizar una compra, son más atractivos para los clientes potenciales que los más complicados. Mortazavi, Rahim Esfindani y Shaemi Barzoki (2014) sugieren que los clientes con facilidad de uso percibida tienden a tener una mayor intención de comprar productos en tiendas virtuales. Por lo tanto, la facilidad de uso relacionada con las funciones e interfaces del sitio web de comercio electrónico es útil para pronosticar la intención de los usuarios hacia la compra electrónica. La facilidad de uso percibida es muy relevante para la manera en que los clientes experimentan las compras en línea de acuerdo con las aplicaciones de Internet, lo que está fuertemente asociado con la intención de compra (Moslehpour, Pham, Wong y Bilgicli, 2018). Si bien estas investigaciones aportan importante conocimiento sobre la relación de los factores del modelo TAM en la intención de compra online, para conocimiento de los autores no existen estudios que hayan analizado estos factores en sitios de redes sociales. Con base en esto se plantean las siguientes hipótesis de investigación:

- H1: La utilidad percibida de los sitios de redes sociales, influyen en la intención de compra en línea de servicios de alojamiento

- H2: La facilidad de uso percibida de los sitios de redes sociales, influyen en la intención de compra en línea de servicios de alojamiento 


\section{Metodología}

El estudio se realizó aplicando una encuesta a una muestra representativa de 382 turistas que se hospedaron en diferentes establecimientos de alojamiento de la ciudad. Se aplicó un muestreo probabilístico estratificado, de acuerdo con las categorías de los establecimientos. Las variables fueron medidas a través del Instrumento propuesto por Rodríguez del Bosque (2008) el cual mide cada una de las variables latentes con cuatro preguntas cada una. Todas las preguntas fueron evaluadas a través de una escala de Likert de uno a cinco.

El análisis de datos y comprobación de hipótesis se realizó a través de modelos de ecuaciones estructurales. En una primera fase se realizó un análisis descriptivo para identificar las características más importantes de los encuestados. Posteriormente se analizaron la confiabilidad y validez de las escalas a través del cálculo del Alpha de Cronbach y Análisis Factorial Confirmatorio respectivamente. Este último análisis permitió la comprobación del ajuste del modelo analizado, así como la validez convergente y divergente. La relación entre las variables latentes se analizó a través del modelo estructural que especifica la relación entra las variables latentes Facilidad de Uso, Utilidad e Intención de Compra, de acuerdo a las hipótesis planteadas. Los datos fueron procesados y analizados con el Software SPSS versión 22 y su complemento AMOS.

\section{Resultados}

\section{Características de los participantes}

De todos los turistas encuestados, el $53.7 \%$ eran mujeres y el $46.3 \%$ hombres. La mayoría correspondiente al $58.4 \%$, reportó estar un rango de edad de 21 a 40 años seguido de un 34.6\% que tiene menos de 20 años. El 43.7\% reporto que navega en internet más de dos horas al día y el $43.2 \%$ lo hace principalmente en las noches. La aplicación principal que utilizan mientras navegan en Internet son las redes Sociales ( $80 \%$ de los encuestados).

\section{Confiabilidad, Validez y Comprobación de Hipótesis}

Con relación a la confiabilidad de las escalas, el análisis realizado a través del cálculo del Alpha de Cronbach arrojó un valor mayor a 0.7 en las escalas que se utilizaron para medir cada una de las variables de estudio. Estos resultados revelan que las escalas utilizadas son confiables y consistentes (Ajzen, 2006). Sin embargo, es importante aclarar que de acuerdo a los resultados del análisis factorial confirmatorio realizado a la variable latente Intención de Compra, fue necesario eliminar uno de los ítems utilizado ya que su carga factorial esta variable latente no fue significativa (Byrne, 2009). En los demás casos no fue necesario realizar ningún ajuste.

El Ajuste del Modelo fue evaluado a través de los índices de ajuste GFI y AGFI, propuestos por Chion y Charles (2016). Los resultados mostraron que el modelo presentó un ajuste aceptable ya que los índices presentaron valores de 0.945 y 0.850 respectivamente. Para considerar un modelo con ajuste aceptable estos índices deben obtener valores mayores a 0.90 y 0.80 
respectivamente. Con respecto a la validez convergente y divergente, ambos criterios fueron alcanzados.

Para la comprobación de hipótesis se utilizó el modelo que se presenta en la Figura 1.

\section{Figura 1}

\section{Modelo estructural analizado}

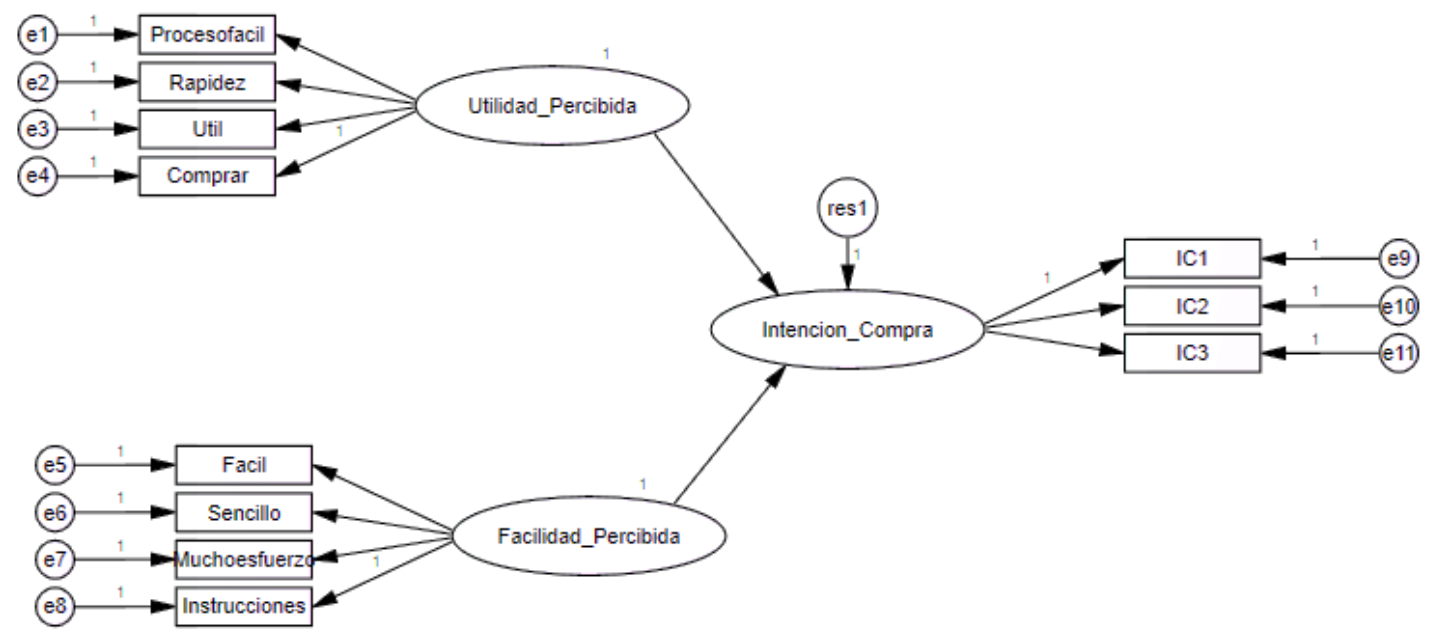

La relación entre utilidad percibida e intención de compra fue negativa y no significativa $(-0.11$; p-valor > 0.05) llevando a rechazar la hipótesis H1. Por otro lado, facilidad de uso percibida tiene una relación positiva y significativa con la intención de compra online $(0.99$; p-valor $<0.05)$ llevando a aceptar la hipótesis de investigación H2.

\section{Conclusiones}

El objetivo principal de la presente investigación fue identificar los principales factores que inciden en la intención de compra de servicios de alojamiento a través de redes sociales. Para ello, se aplicó el modelo de aceptación tecnológica, el cual establece como principales factores que motivan la intención de compra a la utilidad percibida y a la facilidad de uso percibida. El análisis factorial confirmatorio permitió corroborar la adecuación del modelo para analizar este tipo de comportamientos. Por otro lado, los resultados del análisis de las relaciones entre variables llevaron a concluir que únicamente la facilidad de uso percibida influye positiva $\mathrm{y}$ significativamente en la intención de compra de servicios de alojamiento a través de redes sociales, lo cual permite concluir que los gerentes y propietarios de establecimientos de alojamiento deben poner más énfasis en los aspectos hedónicos de facilidad de uso de los sitios web de redes sociales si desean incrementar sus niveles de ventas. No obstante, se deben fortalecer también los aspectos utilitarios de estos espacios virtuales con el propósito de mejorar de alguna manera la intención de compra de los usuarios. 
Los resultados corroboran y contradicen estudio donde se ha analizado el Modelo de Aceptación Tecnológica y la intención de compra en ambientes virtuales. No obstante, para conocimiento de los autores no existen estudios similares que se hayan realizado en entornos de redes sociales. La investigación fue realizada en una muestra significativa de turistas que utilizaron servicios de alojamiento lo cual puede limitar la generalización de los resultados obtenidos. En futuras investigaciones se recomienda ampliar el análisis a otros ámbitos del sector turístico. Por otro lado, la investigación fue de corte transversal lo cual impide identificar cómo evoluciona el fenómeno estudiado a través del tiempo. En futuras investigaciones se podría aplicar estudios de corte longitudinal.

\section{Referencias bibliográficas}

Ajzen, I. (2006). Constructing a TpB questionnaire: conceptual and methodological considerations. Recuperado de: http://www.people.umass.edu/aizen

Ajzen, I. (1991). The theory of planned behavior. Organizational Behavior and Human Decision Processes, 50(2), 179-211. doi:10.1016/0749-5978(91)90020-T

Aldás-Manzano, J., Ruiz-Mafé, C., \& Sanz-Blas, S. (2009). Exploring individual personality factors as drivers of M-shopping acceptance. Industrial Management \& Data Systems, 109(6), 739-757.

Aluri, A. K. (2012). Does embedding social media channels in hotel websites influence travelers' satisfaction and purchase intentions? (Doctoral Dissertation, Oklahoma State University).

Busalim, A., Chen Hussin, A. R., \& Fadhil Yusof, A. (2017). Customer Engagement in Social Commerce: a Theoretical Review, 95(22), 1-68.

Childers, T. L., Carr, C. L., Peck, J., \& Carson, S. (2001). Hedonic and utilitarian motivations for online retail shopping behavior. Journal of retailing, 77(4), 511-535.

Chion, S., \& Charles, V. (2016). Analítica de datos para la modelación estructural. Lima, Perú: Pearson.

Chu, A. Z. C., \& Chu, R. J. C. (2011). The intranet's role in newcomer socialization in the hotel industry in Taiwan-technology acceptance model analysis. The International Journal of Human Resource Management, 22(05), 1163-1179.

Davis, F. D. (1989). Perceived usefulness, perceived ease of use, and user acceptance of information technology. MIS Quarterly, 13, 319-340. doi:10.2307/249008.

Devaraj, S., Easley, R. F., \& Crant, J. M. (2008). Research note-how does personality matter? Relating the five-factor model to technology acceptance and use. Information systems research, 19(1), 93-105.

Fishbein, M., \& Ajzen, I. (1975). Belief, attitude, intention and behavior: An introduction to theory and research. Reading, MA: Addison-Wesley Publisher.

Gefen, D., Karahanna, E., \& Straub, D. W. (2003). Trust and TAM in online shopping: an integrated model. MIS quarterly, 27(1), 51-90.

Hajli, M. (2015). An integrated model for e-commerce adoption at the customer level with the impact of social commerce. International Journal of Information Science and Management (IJISM), 77-97

Javed, M. K., Nazam, M., Ahmad, J., Nadeem, A. H., \& Qadeer, T. (2015). Role of Web in an Online Setting: Consumers perceived risk toward online purchase intention. 
In Proceedings of the Ninth International Conference on Management Science and Engineering Management (pp. 617-625). Springer, Berlin, Heidelberg.

Kim, S., \& Park, H. (2013). Effects of various characteristics of social commerce (s-commerce) on consumers' trust and trust performance. International Journal of Information Management, 33(2), 318-332. https://doi.org/10.1016/j.ijinfomgt.2012.11.006

Kim, H., \& Song, J. (2010). The quality of word-of-mouth in the online shopping mall. Journal of Research in Interactive Marketing, 4(4), 376-390.

Mortazavi, M., Rahim Esfidani, M., \& Shaemi Barzoki, A. (2014). Influencing VSN users' purchase intentions: The roles of flow, trust and eWOM. Journal of Research in Interactive Marketing, 8(2), 102-123.

Memarzadeh, F., Blum, S. \& Adams, Ch. (2016). Business Travelers' Intention to Purchase: The Application of Technology Acceptance Model (TAM). Journal of Quality Assurance in Hospitality and Tourism. 17(4), 412-424.

Moslehpour, M., Pham, V., Wong, W. K., \& Bilgiçli, İ. (2018). E-purchase intention of Taiwanese consumers: Sustainable mediation of perceived usefulness and perceived ease of use. Sustainability, 10(1), 234.

Özbek, V., Alnıaçık, Ü., Koc, F., Akkılıç, M. E., \& Kaş, E. (2014). The impact of personality on technology acceptance: A study on smart phone users. Procedia-Social and Behavioral Sciences, 150, 541-551.

Pavlou, P. A. (2003). Consumer acceptance of electronic commerce: Integrating trust and risk with the technology acceptance model. International journal of electronic commerce, 7(3), 101134.

Pookulangara, S., \& Koesler, K. (2011). Cultural influence on consumers' usage of social networks and its' impact on online purchase intentions. Journal of Retailing and Consumer Services, 18(4), 348-354.

Punnoose, A. C. (2012). Determinants of intention to use eLearning based on the technology acceptance model. Journal of Information Technology Education: Research, 11(1), 301337.

Ramayah, T., Rahman, S. A., \& Ling, N. C. (2018). How do Consumption Values Influence Online Purchase Intention among School Leavers in Malaysia? Revista Brasileira de Gestão de Negócios, 20(4), 638-654.

San Martín, H., \& Herrero, Á. (2012). Influence of the user's psychological factors on the online purchase intention in rural tourism: Integrating innovativeness to the UTAUT framework. Tourism Management, 33(2), 341-350.

Wallace, L. G., \& Sheetz, S. D. (2014). The adoption of software measures: A technology acceptance model (TAM) perspective. Information \& Management, 51(2), 249-259.

Yilmaz, Ö. (2014). The effect of websites on customer preferences related to tourism products within the framework of Technological Acceptance Model (TAM). IIB International Refereed Academic Social Sciences Journal, 5(16), 46.

Yoon, H. S., \& Steege, L. M. B. (2013). Development of a quantitative model of the impact of customers' personality and perceptions on Internet banking use. Computers in Human Behavior, 29(3), 1133-1141.

Zwass, V., \& Kendall, K. (1999). Structure and macro-level impacts of electronic commerce. Emerging Information Technologies: Improving Decisions, Cooperation, and Infrastructure, Sage, Beverly Hills, CA, 289-315. 